\title{
Salivary Gland Infection
}

National Cancer Institute

\section{Source}

National Cancer Institute. Salivary Gland Infection. NCI Thesaurus. Code C78601.

A viral (such as mumps) or bacterial infectious process affecting the salivary gland. The salivary gland becomes enlarged and is often painful. 